Research Article

\title{
Pricing of Margin Call Stock Loan Based on the FMLS
}

\author{
Kaili Xiang, ${ }^{1}$ Peng Hu $\mathbb{D}^{1,2}$ and Xiao Li ${ }^{1}{ }^{1}$ \\ ${ }^{1}$ School of Economic Mathematics, Southwestern University of Finance and economics, Chengdu, China \\ ${ }^{2}$ College of Applied Mathematics, Chengdu University of Information Technology, Chengdu, China
}

Correspondence should be addressed to Xiao Li; 471055395@qq.com

Received 22 April 2020; Accepted 8 June 2020; Published 27 June 2020

Guest Editor: Wenguang Yu

Copyright (c) 2020 Kaili Xiang et al. This is an open access article distributed under the Creative Commons Attribution License, which permits unrestricted use, distribution, and reproduction in any medium, provided the original work is properly cited.

In common stock loan, lenders face the risk that their loans will not be repaid if the stock price falls below loan, which limits the issuance and circulation of stock loans. The empirical test suggests that the log-return series of stock price in the US market reject the normal distribution and admit instead a subclass of the asymmetric distribution. In this paper, we investigate the model of the margin call stock loan problem under the assumption that the return of stock follows the finite moment log-stable process (FMLS). In this case, the pricing model of the margin call stock loan can be described by a space-fractional partial differential equation with a time-varying free boundary condition. We transform the free boundary problem to a linear complementarity problem, and the fully-implicit finite difference method that we used is unconditionally stable in both the integer and fractional order. The numerical experiments are carried out to demonstrate differences of the margin call stock loan model under the FMLS and the standard normal distribution. Last, we analyze the impact of key parameters in our model on the margin call stock loan evaluation and give some reasonable explanation.

\section{Introduction}

Stock loan is a contract that the holders of securities take these securities as collateral to obtain loans from commercial banks. Xia et al. [1] quantified the stock loan, established the mathematical model of the stock loan with infinite maturity by assuming that the logarithm price of risk assets obeys geometric Brownian motion, and opened the door of research on the pricing of the stock loan.

The common stock loan does not have any restrictions on the borrower. When the total price of the stock runs below the loan, the rational borrower will default and the lender will bear the loss, which brings the risk to the lender and reduces the supply and circulation of the stock loan. In order to control the risk, Liang et al. [2] studied the infinite maturity stock loan with automatic termination clause, limit, and additional margin. They found that the unlimited stock loan would make the lender unable to obtain the maximum interest income, and the borrower could not borrow enough funds when he pledged the assets, so that the allocation efficiency of funds could not be maximized. Grasselli and Gómez [3] studied the trading restrictions on the stock holders in the incomplete market. According to the utility function of the fund borrower, the limited maturity stock loan is priced, and the limited maturity stock loan is priced according to the variational inequality. Wong [4] studied the optimal stop time problem of the infinite maturity stock loan similar to a permanent American option driven by the index Levy process and uses variational inequality to solve the problem. Cai and Sun [5] studied the infinite maturity and limited maturity stock loan models under the super index jump diffusion model. The accuracy of the solution is proved by numerical examples. Wong et al. [6] studied the case that stock volatility is a stochastic process. When the interest rate is negative, the optimal stop time of an American call option is considered.

Through the research and empirical test, scholars found that the classical B-S framework does not conform to the actual situation, and the logarithmic distribution of risk asset prices often has a "peak thick tail" phenomenon, which is consistent with the fact that the stock price is an autocorrelation process. Carr et al. [7] studied the volatility smile of asset prices through empirical research and found that the FMLS process model can better describe asset price changes. 
As a special Levy process, the FMLS process can well reflect the large jump of risk asset price and the situation that the logarithm distribution of risk asset price is often biased. In the framework of FMLS, the $\alpha$ steady-state process is used as the driving process to describe the autocorrelation of asset price changes and ensure the stability. When $\alpha<2$, the FMLS model is in good agreement with the phenomenon that the logarithm distribution of risk asset prices has thick tail. Because of its good properties, finite moment log steady state processes are more and more used to simulate asset price movement. $\mathrm{Yu}$ et al. [8] derived the equations and the boundary conditions satisfied by the Gerber-Shiu function, the expected discounted capital injection function and the expected discounted dividend function by assuming that the observation interval and claim amount are exponentially distributed, respectively. Yu et al. [9] considered a class of social optimal mean field control problem of the population growth model. Zhang et al. [10] focused on valuation of the products with guaranteed minimum death benefit (GMDB). The benefit amount is linked to the performance of the underlying asset, which is modeled by an exponential Levy process. Yu et al. [11] used the Fourier cosine series expansion (COS) method to value the guaranteed minimum death benefit (GMDB) products. Peng et al. [12] modeled the insurance company's surplus flow by a perturbed compound Poisson model.

Under the finite moment log-stable (FMLS) model, the partial differential equation of option pricing is not easy to get because of a fractional operator. Cartea [13] first gave the proper partial differential equation of European option pricing under the framework of FMLS by the Fourier transform. Chen et al. [14] gave the analytical solution of European option under the framework of FMLS through the Fourier integral transform on the basis of Cartea, and they studied the numerical solution of an American call option pricing under the framework of FMLS through the prediction correction method [15], in which the moving boundary problem was transformed into the fixed boundary problem through coordinate transformation. Zhang et al. [16] studied the numerical solution of European double barrier options driven by the Levy process, including FMLS, using the second-order implicit difference scheme. Chen [17] studied the second-order finite difference scheme and obtained the numerical solution of the American option in the form of penalty function.

Based on the previous research, we found that under the framework of FMLS, nobody studied the margin call stock loan. In the actual economic activities, the demand for the margin stock loan is increasing constantly.

The rest of this paper is arranged as follows. In the Section 2, we establish the mathematical model of the stock loan with margin call, transform the moving boundary problem into the fixed boundary problem through coordinate transformation, and finally transform it into the linear complementarity problem. In Section 3, we obtain the numerical solution of the linear complementarity problem by the finite difference method. In the Section 4 , the validity of the numerical solution and the parameter analysis are verified by numerical examples. Finally, we draw the conclusion in the Section 5.

\section{Pricing Model}

The margin call stock loan: the borrower pledged stock $S$ and got loan $K$ from lender. The borrower can choose to repay the loan at any time. However, the borrower needs to pay a certain amount of interest while paying the loan $K$, so the borrower chooses the repayment amount at time $t$ as $\mathrm{Ke}^{\gamma t}$ when $S_{t}<K e^{\gamma t}$; rational people will not repay, and his payment at time $t$ is $\left(S_{t}-K e^{\gamma t}\right)^{+}$. In order to control the risk, the margin call stock loan has set a lower bound $S_{B}$; when the stock price $S_{t}$ at $t$ moment runs below $S_{B}$ (generally $S_{B}=K e^{\gamma t}$ ), the lender have the right to require the borrower to recover the funds of $\Delta K e^{\gamma t_{q}}$, where $\Delta$ is the share to be recovered, and then, the remaining loan is $(1-\Delta) K e^{\gamma t}$. After the payment is made, the margin call stock loan becomes a nonrecourse stock loan with a maturity of $T-t$; therefore, the value of the stock loan after recovery is $R(t)=V\left(x_{B}, 0 ;(1-\Delta) K e^{\gamma t}\right)-\Delta K e^{\gamma t}$, where $V$ is the value of the nonrecourse stock loan with a maturity of $T-t_{q}$ and strike price of $(1-\Delta) \mathrm{Ke}^{\gamma t}$.

The margin call stock loan stipulates a lower bound of the stock price. When the stock price reaches the lower bound, the stock loan is suspended, and the lender has the right to require the borrower to recover part of the loan. After the borrower recovers the loan, the stock loan with recourse is converted into a stock loan without recourse until the maturity date.

In conclusion, there are two stages in the margin call stock loan: the stage with recourse and the stage without recourse. This is similar to an American call option with a change barrier in the first stage and an American call option with a change strike price in the second stage, as shown in Figure 1.

The value of the margin call stock loan is $V_{\mathrm{mc}}$ (margin call [18]), where $V$ is the nonrecourse stock loan. Let $x_{t}=\ln S_{t}$, and under the risk neutral measure, the stock price under the framework of FMLS obeys the following stochastic differential equation:

$$
\mathrm{d} x_{t}=(r-D-a) \mathrm{d} t+\sigma \mathrm{d} L_{t}^{\mathrm{FMLS}},
$$

where $r$ is the risk-free interest rate, $D$ is the continuous dividend rate of stock, $\sigma$ is volatility, $\mathrm{d} L_{t}^{\mathrm{FMLS}}$ is the $\alpha$-steady state stochastic process, $\mathrm{d} L_{t}^{\mathrm{FMLS}} \sim L^{\alpha}\left(0, \mathrm{~d} t^{1 / \alpha},-1\right), a$ is the convex adjustment, and $a=-\sigma^{\alpha} \sec (\alpha \pi / 2)$. According to the above assumptions, the partial differential equation and boundary conditions of the pricing of the margin call stock loan can be obtained as

$$
\begin{aligned}
\frac{\partial V_{\mathrm{mc}}(x, t)}{\partial t}= & r V_{\mathrm{mc}}(x, t)-(r-D-a) \frac{\partial V_{\mathrm{mc}}(x, t)}{\partial x} \\
& -a_{-\infty} D_{x}^{\alpha} V_{\mathrm{mc}}(x, t) .
\end{aligned}
$$

Boundary condition 1: terminal condition.

$$
V_{\mathrm{mc}}(x, T)=\left(e^{x_{T}}-K e^{\gamma T}\right)^{+} .
$$

Boundary condition 2: when $S_{t}=S_{B}=K e^{\gamma t}$, i.e., $x_{t}=x_{B}$, the borrower needs to recover $\Delta K e^{\gamma t}$, where 


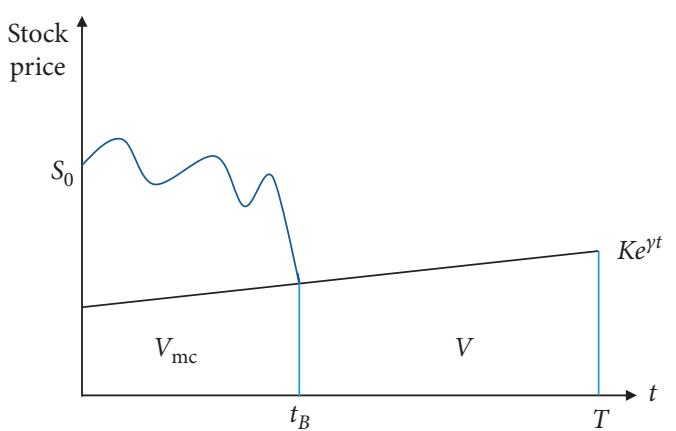

Figure 1: Two stages of the margin call stock loan.

$V$ is the nonrecourse stock loan with the execution price of $(1-\Delta) K e^{\gamma t}$ and the maturity date of $T-t_{B}$. So,

$R(t)=V_{\mathrm{mc}}(x, t)=V\left(x, 0 ;(1-\Delta) K e^{\gamma t}, T-t_{B}\right)-\Delta K e^{\gamma t}$.

In order to reduce the error of fractional order numerical calculation, take

$$
\begin{aligned}
R(x, t)= & V_{\mathrm{mc}}(x, t)=V\left(x, 0 ;(1-\Delta) K e^{\gamma t}, T-t_{B}\right) \\
& -\Delta K e^{\gamma t}, \quad x \in\left(-\infty, x_{B}\right) .
\end{aligned}
$$

When $\Delta=0$, the margin call stock loan becomes a stock loan without recourse.

Boundary condition 3: when the stock price approaches the free boundary,

$$
V_{\mathrm{mc}}\left(x_{f}, t\right)=\left(e^{x_{f}}-K e^{\gamma t}\right)^{+} .
$$

Boundary condition 4 : when the stock price approaches the free boundary, it should be kept smooth.

$$
\frac{\partial V_{\mathrm{mc}}\left(x_{f}, t\right)}{\partial x}=e^{x_{f}} .
$$

In conclusion, the partial differential equations and boundary conditions of the nonrecourse stock loan pricing with variable strike price under the framework of FMLS are as follows:

$$
\left\{\begin{array}{l}
\frac{\partial V_{\mathrm{mc}}(x, t)}{\partial t}=r V_{\mathrm{mc}}(x, t)-(r-D-a) \frac{\partial V_{\mathrm{mc}}(x, t)}{\partial x}-a_{-\infty} D_{x}^{\alpha} V_{\mathrm{mc}}(x, t), \quad x \in\left(-\infty, x_{f}\right), t \in[0, T], 1<\alpha<2 \\
V_{\mathrm{mc}}(x, t)=R(x, t), \quad x \in\left(-\infty, x_{B}\right), \\
V_{\mathrm{mc}}(x, T)=\left(e^{x_{T}}-K e^{\gamma T}\right)^{+} \\
\frac{\partial V_{\mathrm{mc}}\left(x_{f}, t\right)}{\partial x}=e^{x_{f}}
\end{array}\right.
$$

Coordinate transformation for $(8)$

$$
\left\{\begin{array}{l}
y_{t}=x_{t}-\gamma t, \\
\tau=T-t, \\
\bar{V}(y, \tau)=e^{-\alpha t} V(x, t) .
\end{array}\right.
$$

The final value problem with moving boundary is transformed into the initial boundary value problem with a fixed boundary.

First, the coordinate transformation of the fractional order operator is

$$
\begin{aligned}
{ }_{-\infty} D_{x}^{\alpha} V_{\mathrm{mc}}(x, t) & =\frac{1}{\Gamma(n-\alpha)} \frac{\mathrm{d}^{n}}{\mathrm{~d} x_{t}^{n}} \int_{-\infty}^{x_{t}} \frac{V_{\mathrm{mc}}(z, t)}{\left(x_{t}-z\right)^{\alpha-n+1}} \mathrm{~d} z \\
& =\frac{1}{\Gamma(n-\alpha)} \frac{\mathrm{d}^{n}}{\mathrm{~d} x_{t}^{n}} \int_{-\infty}^{x_{t}} \frac{e^{\alpha t} \bar{V}_{\mathrm{mc}}(y, \tau)}{\left(x_{t}-z\right)^{\alpha-n+1} \mathrm{~d} z} \\
& =\frac{e^{\alpha t}}{\Gamma(n-\alpha)} \frac{\mathrm{d}^{n}}{\mathrm{~d}\left(y_{t}+\gamma t\right)^{n}} \int_{-\infty}^{x} \frac{\bar{V}_{\mathrm{mc}}(y, \tau)}{\left(y_{t}+\gamma t-z\right)^{\alpha-n+1}} \mathrm{~d} z \\
& =\frac{e^{\alpha t}}{\Gamma(n-\alpha)} \frac{\mathrm{d}^{n}}{\mathrm{~d} y_{t}^{n}} \int_{-\infty}^{y_{t}} \frac{\bar{V}_{\mathrm{mc}}(y, \tau)}{\left(y_{t}-z\right)^{\alpha-n+1}} \mathrm{~d} z,
\end{aligned}
$$




$$
\left\{\begin{array}{l}
\frac{\partial \bar{V}_{\mathrm{mc}}(y, \tau)}{\partial \tau}=(r-\gamma-D-a) \frac{\partial \bar{V}_{\mathrm{mc}}(y, \tau)}{\partial y}+a_{-\infty} D_{y}^{\alpha} \bar{V}_{\mathrm{mc}}(y, \tau)-(r-\gamma) \bar{V}_{\mathrm{mc}}(y, \tau), \quad y \in\left(-\infty, y_{f}\right], \tau \in[0, T], 1<\alpha<2 \\
\bar{V}_{\mathrm{mc}}(y, 0)=\left(e^{y_{0}}-K\right)^{+} \\
\bar{V}_{\mathrm{mc}}(y, \tau)=\bar{V}(y, \tau ;(1-\Delta) K, \tau)-\Delta K, \quad y \in\left(-\infty, y_{B}\right] \\
\bar{V}_{\mathrm{mc}}\left(y_{f}, \tau\right)=\left(e^{y_{f}}-K\right)^{+} \\
\frac{\partial \bar{V}_{\mathrm{mc}}\left(y_{f}, \tau\right)}{\partial y}=e^{y_{f}}
\end{array}\right.
$$

Next, we transform (11) from the free boundary problem into a linear complementarity problem:

$$
\left\{\begin{array}{l}
\min \left(\frac{\partial \bar{V}_{\mathrm{mc}}}{\partial \tau}-L_{y} \bar{V}_{\mathrm{mc}}, \bar{V}_{\mathrm{mc}}-\left(e^{y}-K\right)^{+}\right)=0, \\
y \in R, \tau \in[0, T], \\
\bar{V}_{\mathrm{mc}}(y, 0)=\left(e^{y_{0}}-K\right)^{+}, \quad y>y_{B}, \\
\bar{V}_{\mathrm{mc}}(y, \tau)=\bar{V}(y, \tau ;(1-\Delta) K, \tau)-\Delta K, \\
y \in\left(-\infty, y_{B}\right], \tau \in[0, T],
\end{array}\right.
$$

where

$$
\begin{aligned}
L_{y} \bar{V}_{\mathrm{mc}}= & (r-\gamma-D-a) \frac{\partial \bar{V}_{\mathrm{mc}}(y, \tau)}{\partial y} \\
& +a_{-\infty} D_{y}^{\alpha} \bar{V}_{\mathrm{mc}}(y, \tau)-(r-\gamma) \bar{V}_{\mathrm{mc}}(y, \tau) .
\end{aligned}
$$

So far, we derive the partial differential equations and boundary conditions for pricing contracts with recourse under the framework of FMLS, where the lower boundary is relatively special due to the limitation of recourse. Then, the final value problem with variable boundary is transformed into the initial value problem with fixed boundary by coordinate transformation. Finally, the free boundary problem is transformed into a linear complementarity problem, and a simpler and more convenient linear complementarity problem is obtained. In the next section, we will use the finite difference scheme to give the numerical solution of equation (12).

\section{Numerical Format of the Model}

Before numerical calculation, it is necessary to establish grid and approximate PDE. First, we divide the domain. Suppose $y_{B}$ is the ordinate corresponding to the barrier price. Let $0 \leq \tau=n \Delta \tau \leq T, n=0,1, \ldots, N ; L \leq y \leq R, \Delta y=\left(R-y_{B} /\right.$ $M), y_{m}=L+i \Delta y, \quad m=0,1, \ldots, M$; and $\bar{V}_{\mathrm{mc}}^{\mathrm{n}} \approx \bar{V}_{\mathrm{mc}}\left(y_{m}\right.$, $\left.\tau_{n}, \alpha\right)$, and $y_{B}=L+b \Delta y$ is the ordinate corresponding to the margin call boundary.
The initial value and boundary conditions are as follows. Initial value condition:

$$
\bar{V}_{\mathrm{mc}_{m}}^{0}=\left(e^{y_{m}}-K\right)^{+}, \quad m=b, 1, \ldots, M .
$$

Boundary condition 1 :

$$
\begin{array}{r}
\bar{V}_{\mathrm{mc}_{m}}^{n}=\bar{V}\left(y_{m}, \tau_{n} ;(1-\Delta) K, \tau_{n}\right)-\Delta K, \\
n=0,1, \ldots, N, m=0,1, \ldots, b .
\end{array}
$$

Boundary condition 2:

$$
\bar{V}_{\mathrm{mc}_{M}}^{n}=e^{y_{m}}-K, \quad n=0,1, \ldots, N .
$$

We use the central difference scheme to approximate the first-order time and space partial derivatives:

$$
\begin{aligned}
& \frac{\partial \bar{V}_{\mathrm{mc}}\left(y_{m}, \tau_{n}\right)}{\partial \tau}=\frac{\bar{V}_{\mathrm{mc}_{m}}^{n+1}-\bar{V}_{\mathrm{mc}_{\mathrm{m}}}^{\mathrm{n}}+O(\Delta \tau),}{\Delta \tau}+ \\
& \frac{\partial \bar{V}_{\mathrm{mc}}\left(y_{m}, \tau_{n}\right)}{\partial y}=\frac{\bar{V}_{\mathrm{mc} m+1}^{n+1}-\bar{V}_{m-1}^{n+1}}{2 \Delta y}+O\left(\Delta y^{2}\right) .
\end{aligned}
$$

We use the modified Grunwald-Letnikov formula to approximate the space-fractional derivative [19]:

$$
{ }_{-\infty} D_{y}^{\alpha} \bar{V}_{m c}\left(y_{m}, \tau_{n}\right)=\frac{1}{\Delta y^{\alpha}} \sum_{k=0}^{m+1} w_{k}^{(\alpha)} \bar{V}_{m c_{m-k+1}^{n+1}}^{n+O}\left(\Delta y^{2}\right)
$$

where

$$
\begin{aligned}
w_{k}^{(\alpha)} & =(-1)^{k}\left(\begin{array}{l}
\alpha \\
k
\end{array}\right)=\frac{(-1)^{k} \Gamma(\alpha+1)}{\Gamma(k+1) \Gamma(\alpha-k+1)} \\
& =\frac{\alpha(\alpha-1) \cdots(\alpha-k+1)}{k !}
\end{aligned}
$$

is called the Grunwald-Letnikov coefficient. In numerical calculation, in order to save memory, the iterative algorithm is generally used to calculate $w_{k}^{(\alpha)}$. From the above formula, we found that the fractional derivative is related to all the values before $m+1$, which means the fractional derivative is 
a nonlocal operator, which can well explain that the underlying asset price movement is related to the previous historical price.
We get the implicit difference scheme of equation (11) based on the modified Grunwald-Letnikov formula approximation:

$$
\begin{aligned}
& \frac{\bar{V}_{\mathrm{mc}_{m}}^{n+1}-\bar{V}_{\mathrm{mc}_{m}}^{n}}{\Delta \tau}=\frac{1}{2}(r-\gamma-D-a)\left(\frac{\bar{V}_{\mathrm{mc}_{m+1}}^{n+1}-\bar{V}_{\mathrm{mc}_{m-1}}^{n+1}}{2 \Delta y}+\frac{\bar{V}_{\mathrm{mc}_{m+1}}^{n+1}-V_{\mathrm{mc}_{m-1}}^{n+1}}{2 \Delta y}\right) \\
& +\frac{1}{2} a\left(\frac{1}{\Delta y^{\alpha}} \sum_{k=0}^{m+1} w_{k}^{(\alpha)} \bar{V}_{\mathrm{mc}_{m-k+1}^{n+1}}+\frac{1}{\Delta y^{\alpha}} \sum_{k=0}^{m+1} w_{k}^{(\alpha)} \bar{V}_{\mathrm{mc}_{m-k+1}}^{n+1}\right) \\
& -\frac{1}{2}(r-\gamma)\left(\bar{V}_{\mathrm{mc}_{m}}^{n+1}+V_{\mathrm{mc}_{m}}^{n}\right) .
\end{aligned}
$$

In reference [19], the scheme has been proved to be unconditionally stable. Equation (20) is sorted out as follows:

$$
\begin{aligned}
& -h_{1} \sum_{k=3}^{m+1} w_{k}^{(\alpha)} \bar{V}_{\mathrm{mc}_{m-k+1}+1}^{n+1}+\left(h_{2}-h_{1} w_{2}^{(\alpha)}\right) \bar{V}_{\mathrm{mc}_{m-1}}^{n+1}+\left(1-h_{1} w_{1}^{(\alpha)}+h_{3}\right) \bar{V}_{\mathrm{mc}_{m}}^{n+1}-\left(h_{1} w_{0}^{(\alpha)}+h_{2}\right) \bar{V}_{\mathrm{mc}_{m+1}}^{n+1} \\
= & h_{1} \sum_{k=3}^{m+1} w_{k}^{(\alpha)} \bar{V}_{\mathrm{mc}_{m-k+1}}^{n}+\left(h_{1} w_{2}^{(\alpha)}-h_{2}\right) \bar{V}_{\mathrm{mc}_{m-1}}^{n}+\left(1+h_{1} w_{1}^{(\alpha)}-h_{3}\right) \bar{V}_{\mathrm{mc}_{m}}^{n}+\left(h_{1} w_{0}^{(\alpha)}+h_{2}\right) \bar{V}_{\mathrm{mc}_{m+1}}^{n},
\end{aligned}
$$

where

$$
\begin{aligned}
& h_{1}=\frac{1}{2} \frac{a \Delta \tau}{\Delta y^{\alpha}}, \\
& h_{2}=\frac{1}{4}(r-\gamma-D-a) \frac{\Delta \tau}{\Delta y}, \\
& h_{3}=\frac{1}{2}(r-\gamma) \Delta \tau .
\end{aligned}
$$

Write (21) in matrix:

$$
\mathbf{A} \bar{V}_{\mathrm{mc}}^{\mathbf{n}+\mathbf{1}}=\mathbf{B} \bar{V}_{\mathrm{mc}}^{\mathbf{n}},
$$

where

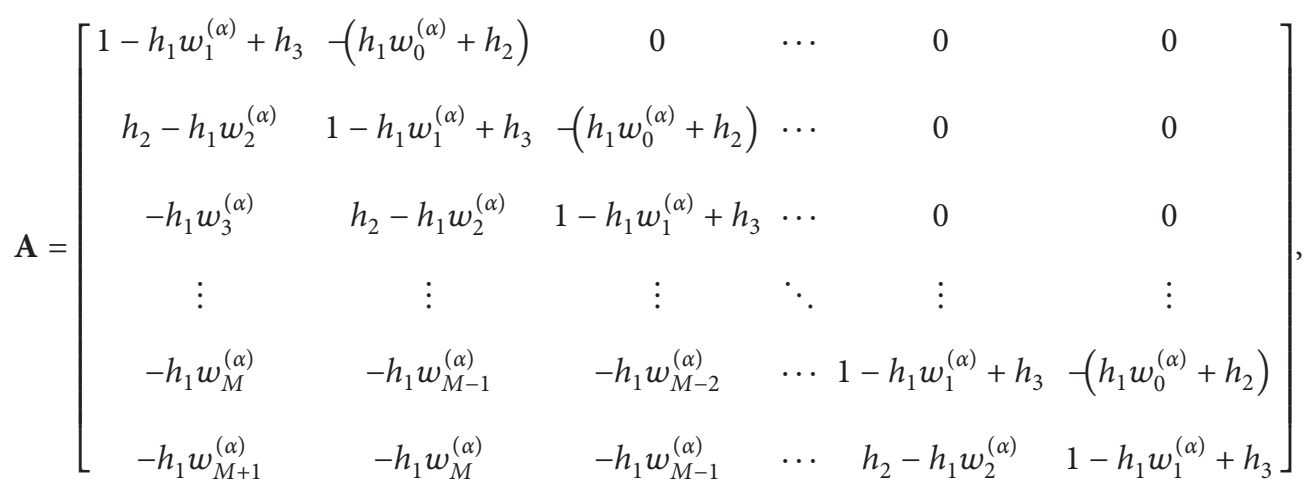




$$
\begin{aligned}
& \mathbf{B}=\left[\begin{array}{cccccc}
1+h_{1} w_{1}^{(\alpha)}-h_{3} & h_{1} w_{0}^{(\alpha)}+h_{2} & 0 & \cdots & 0 & 0 \\
h_{1} w_{2}^{(\alpha)}-h_{2} & 1+h_{1} w_{1}^{(\alpha)}-h_{3} & h_{1} w_{0}^{(\alpha)}+h_{2} & \cdots & 0 & 0 \\
h_{1} w_{3}^{(\alpha)} & h_{1} w_{2}^{(\alpha)}-h_{2} & 1+h_{1} w_{1}^{(\alpha)}-h_{3} & \cdots & 0 & 0 \\
\vdots & \vdots & \vdots & \ddots & \vdots & \vdots \\
h_{1} w_{M}^{(\alpha)} & h_{1} w_{M-1}^{(\alpha)} & h_{1} w_{M-2}^{(\alpha)} & \cdots & 1+h_{1} w_{1}^{(\alpha)}-h_{3} & h_{1} w_{0}^{(\alpha)}+h_{2} \\
h_{1} w_{M+1}^{(\alpha)} & h_{1} w_{M}^{(\alpha)} & h_{1} w_{M-1}^{(\alpha)} & \cdots & h_{1} w_{2}^{(\alpha)}-h_{2} & 1+h_{1} w_{1}^{(\alpha)}-h_{3}
\end{array}\right], \\
& \bar{V}_{m c}^{n+1}=\left[\bar{V}_{0}^{n+1}, \bar{V}_{1}^{n+1}, \bar{V}_{2}^{n+1}, \ldots, \bar{V}_{M}^{n+1}\right] \text {, } \\
& \bar{V}_{m c}^{n}=\left[\bar{V}_{0}^{n}, \bar{V}_{1}^{n}, \bar{V}_{2}^{n}, \ldots, \bar{V}_{M}^{n}\right] .
\end{aligned}
$$

Since $B \bar{V}_{\mathrm{m} \in}^{n}$ is known in numerical calculation, we can make $q^{n}=B \bar{V}_{\mathrm{mc}}^{n}$, that is, $A \bar{V}_{\mathrm{mc}}^{n+1}=q^{n}$. It can be obtained by
Gauss elimination $\left[\mathbf{A} \mid \mathbf{q}^{n}\right] \longrightarrow\left[\mathbf{A}^{\prime} \mid \mathbf{q}^{n^{\prime}}\right]$, where $\left[\mathbf{A}^{\prime} \mid \mathbf{q}^{n^{\prime}}\right]$ is the simplest step matrix, so we get

$$
\begin{cases}\bar{V}_{\mathrm{mc}_{m}}^{n+1}=\frac{q_{m}^{n^{\prime}}}{A_{m, m}^{\prime}}, & m=M, \\ \bar{V}_{\mathrm{mc}_{m}^{n+1}}^{n+}=\frac{q_{m}^{n^{\prime}}-A_{m, m+1}^{\prime} \bar{V}_{m+1}^{n+1}}{A_{m, m}^{\prime}}, & m=M-1, M-2, \ldots, b .\end{cases}
$$

Formula (12) and the stock loan have their own characteristics. Formula (20), (21), and (25) are equivalent, and they are only established in area $m=0,1, \ldots, m_{f}$, where $m_{f}$ is the point corresponding to the asset price closest to the free boundary. Obviously, $m_{f}$ is a positive integer less than $M$. Due to the fact that the stock loan can be repaid at any time, obviously $\bar{V}_{m}^{n+1}$ should be greater than or equal to $\left(e^{y_{f}}-K\right)^{+}$. Therefore, the value of the stock loan on each approximation grid point should be expressed as

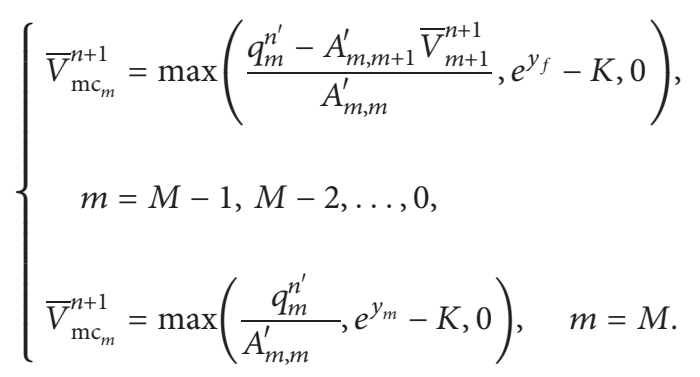

According to the conclusion of reference [20], backward calculation $\bar{V}_{\mathrm{mc}_{m}}^{n+1}$ in (26), it will be more accurate to calculate the value of the stock loan from $M$. Because backward calculation defaults $\bar{V}_{\mathrm{mc}_{m}}^{n+1}=\left(e^{y_{m}}-K\right)^{+}$when $m \geq m_{f}$. When $m<m_{f}$, the value of stock loan is determined by partial differential equation (11).

\section{Numerical Example}

4.1. Model Validation. Generally, the pledge rate of the stock loan is less than 0.6. We set the parameters of stock loans with recourse as follows: initial stock price $S_{0}=100$, loan $K=60$, expiration date $T=3$, risk free interest rate $r=0.05$, dividend rate $D=0.1$, volatility $\gamma=0.1$, tail index $\alpha=1.5$, and recovery factor $\Delta=0.4$, and let $S_{\max }=150, S_{B}=K e^{\gamma t}$. Due to $y_{t}=\ln S_{t}-\gamma t, S_{t}=e^{y_{t}+\gamma t}$, corresponding $y_{\max }=5$ and $y_{B}=4.094$. If $S_{t}$ is used as the boundary, it will cause the boundary moving, so we use the final result $\bar{V}_{m c}$ of model (11) as the inverse coordinate transformation to get $V$, $V_{\mathrm{mc}}(x, t)=e^{\alpha(T-t)} \bar{V}_{\mathrm{mc}}(y, \tau)$, and $e^{\alpha(T-\tau)}$ is always bigger than 1 .

Figure 2 shows that the margin call stock loan is cut off at the barrier price $y_{B}$. It can be seen from the lower boundary, the longer the maturity, the greater the value of the nonrecourse stock loan, and the greater the value of barrier $y_{B}$, the higher the corresponding function image. Under the price of $y_{B}$, the margin call stock loan is transformed into a stock loan without recourse. In this paper, we mainly discuss the nature of the margin call stock loan through numerical experiments.

Figure 3 shows that when the pledge rate $\Delta=0$, margin call stock loans equals no recourse stock loans.

4.2. Parameter Analysis. The risk of the margin call stock loan is more controllable for the lender, so the lender is more willing to improve the pledge rate to borrow more money and obtain more interest. With the increase of pledge rate, the borrower also gets more funds in the controllable range, which improves the allocation efficiency of funds.

Figure 4 shows the impact of different recourse shares $\Delta$ on the value of the margin call stock loan. The right side of the 


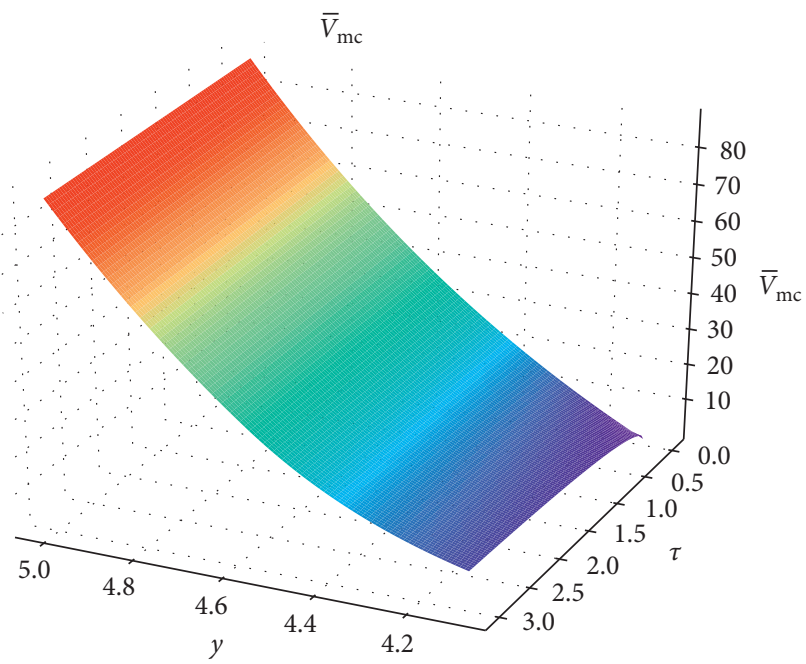

Figure 2: The value of $\bar{V}_{\mathrm{mc}}$.

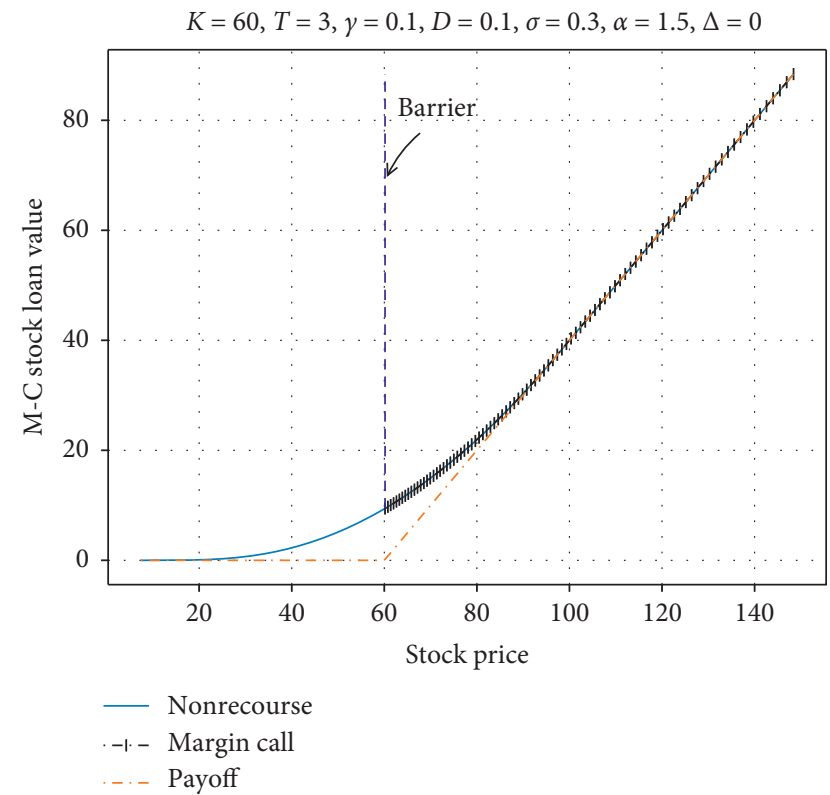

Figure 3: When $\Delta=0, V_{\mathrm{mc}}$ coincides with $V$.

barrier indicates the contract value of the margin call stock loan. The left side of the obstacle is $V(x, 0 ;(1-\Delta) K, T)-\Delta K$, which means, the value of the nonrecourse stock loan with a maturity of $T$ and loan amount of $(1-\Delta) K$ minus the value of recourse currency at time 0 . Figure shows that the lower the share of recourse, the higher the contract value of the stock pledge loan with recourse, which is confirmed by the actual economic activities. And from formula (4), no matter how high the recourse share $\Delta$ is, the contract value of the margin call stock loan is always bigger than zero at the obstacle.

Figure 5 shows the effect of different loan interest rates $\gamma$ on the contract value of the margin call stock loan under the condition that other parameters remain unchanged. We found that the higher the loan interest rate $\gamma$ is, the lower the contract value of the margin call stock loan is because in practice, the higher the loan interest rate is, the higher the interest the borrower needs to pay and the faster the barrier

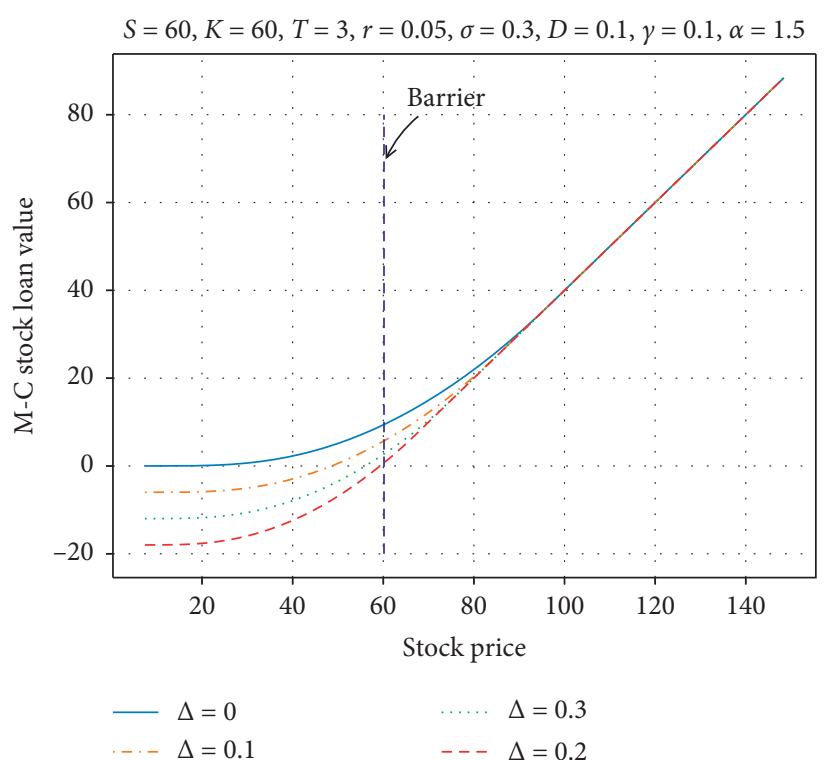

Figure 4: Effect of different recourse shares $\Delta$ on $V_{\mathrm{mc}}$.

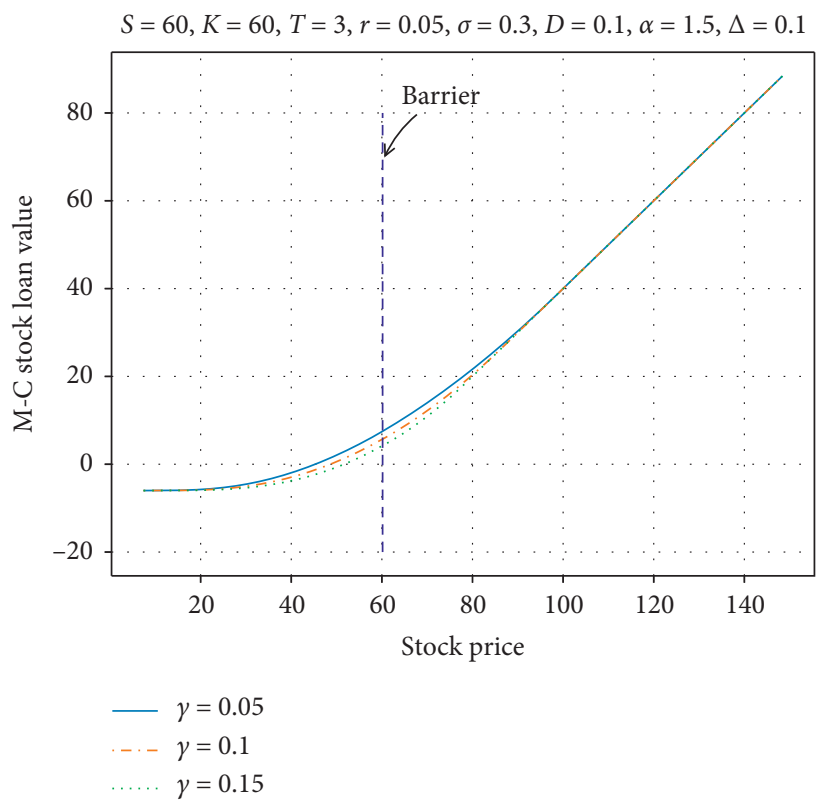

Figure 5: Effect of different loan interest rates $\gamma$ on $V_{\mathrm{mc}}$.

price will rise, so the corresponding contract value of the margin call stock loan will be lower.

Figure 6 shows the effect of different maturity dates $T$ on the contract value of the margin call stock loan under the condition that other parameters remain unchanged. This is because in the actual economic activities, on the one hand, due to the existence of volatility, the longer the time is, the higher the stock price is likely to reach; on the other hand, the loan interest rate and dividend rate are greater than the riskfree interest rate, so the longer the time is, the greater the risk the fund lender bears. In conclusion, the longer the maturity $T$ is, the higher the value of the margin call stock loan is.

Figure 7 shows the effect of different volatility $\sigma$ on the margin call stock loan under the condition that other 


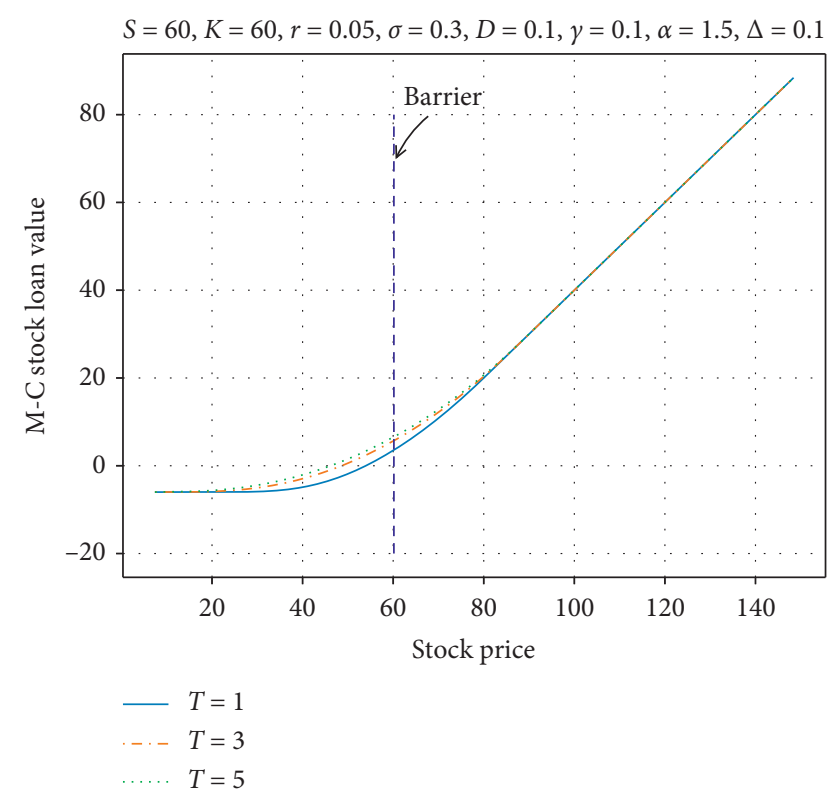

FIgURE 6: Effect of different maturity dates $T$ on $V_{\mathrm{mc}}$.

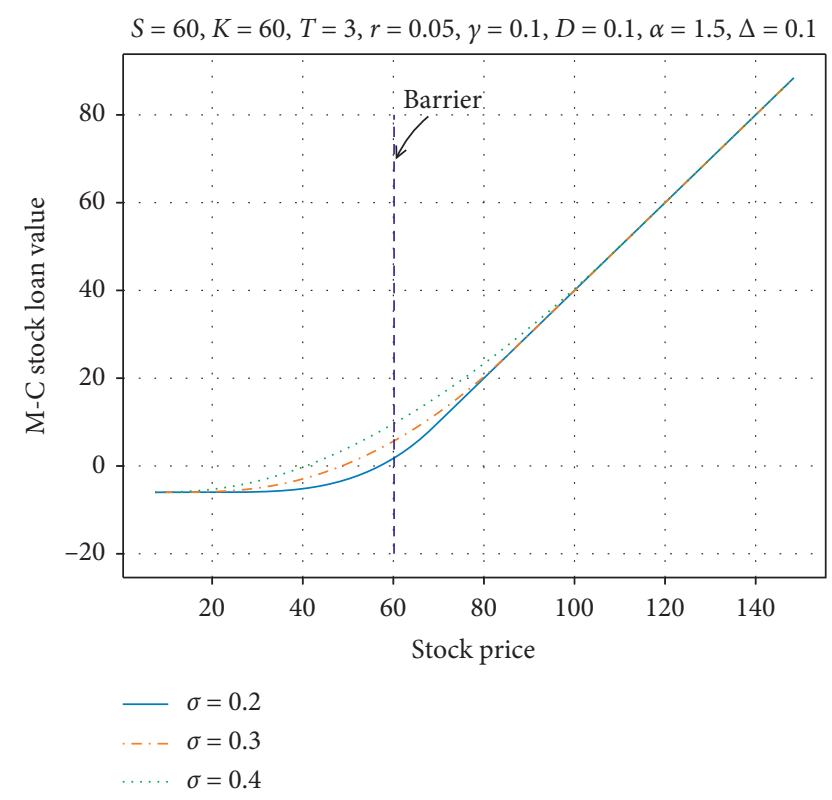

FIGURE 7: Effect of different volatility $\sigma$ on $V_{\mathrm{mc}}$.

parameters are unchanged. It can be seen from the figure that the margin call stock loan increases with the volatility $\sigma$ because the nonrecourse stock loan is similar to an American option. Due to the increase in the value of the American option with the increase of volatility $\sigma$, the increase of volatility $\sigma$ will give a higher initial value to the recourse stock loan. On the other hand, the margin call stock loan is similar to an American barrier option, and the larger the volatility $\sigma$, the higher the value of the margin call stock loan.

Figure 8 shows the effect of different risk-free interest rates $r$ on the contract value of the margin call stock loan under the condition that other parameters remain

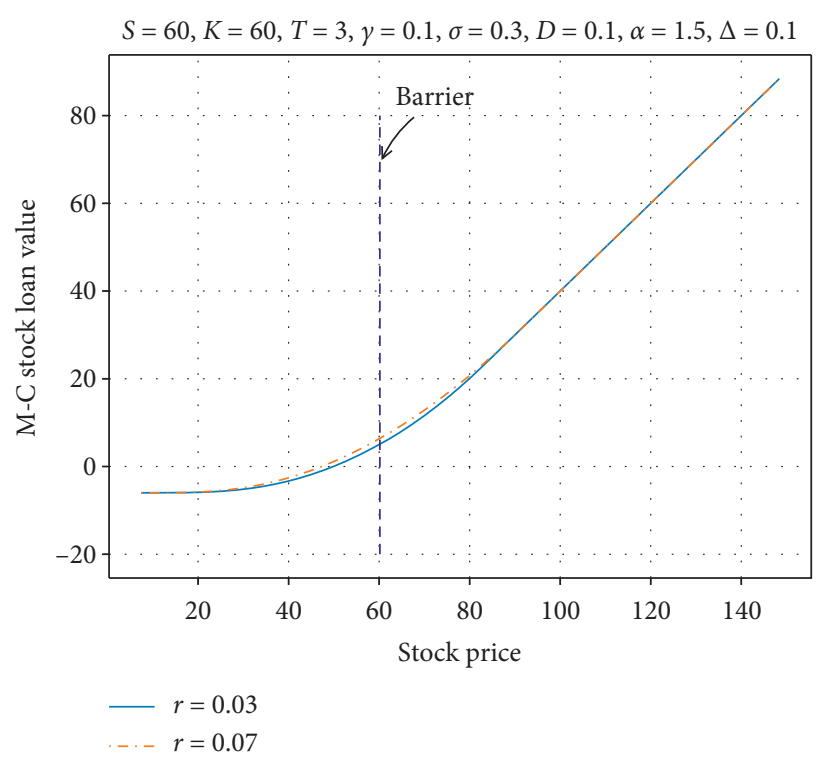

Figure 8: Effect of different risk-free interest rates $r$ on $V_{\text {mc }}$.

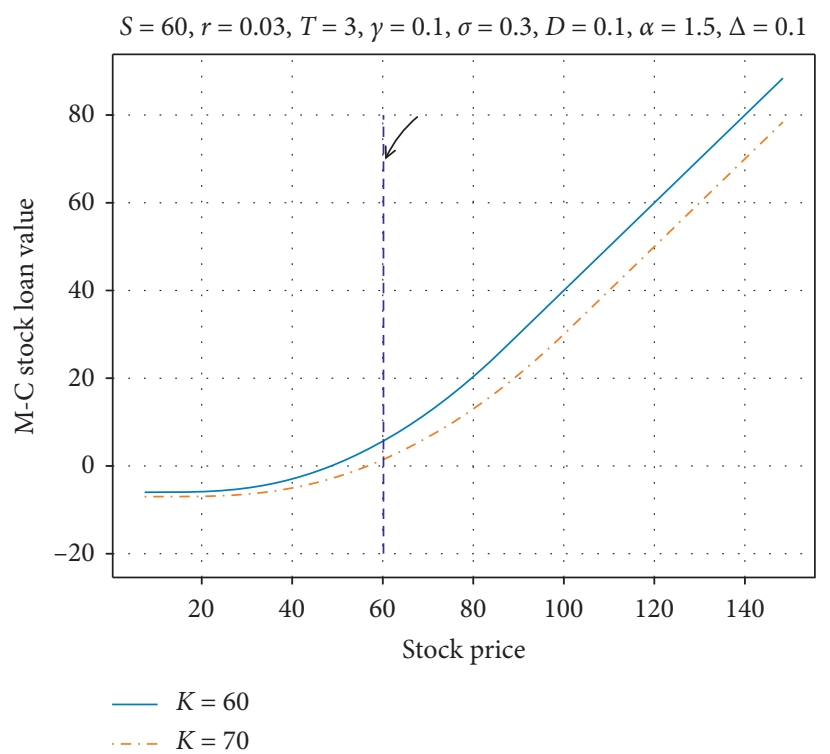

Figure 9: Effect of different loan amounts $K$ on $V_{\mathrm{mc}}$.

unchanged. The figure shows that the value of the margin call stock loan increases with the increase of the risk-free interest rate $r$. Which means the higher the risk-free interest rate is, the higher the contract value of the margin call stock loan is.

Because the risk of the margin call stock loan is more controllable for the lender, in order to obtain more interest income, the lender is willing to increase the pledge rate, which means to provide more loans. Figure 9 shows the effect of different loan amounts $K$ on the contract value of the margin call stock loan. Because the higher the loan amount is, the easier the stock price is to reach the lower bound, the lower the contract value of the corresponding margin call stock loan is. 


\section{Conclusion}

In this paper, we analyze the pricing process of the margin call stock loan under the framework of FMLS. We take the value of the nonrecourse stock loan as a boundary condition of the barrier of the recourse stock loan and study the pricing of the recourse stock loan. The partial differential equations and boundary conditions for the pricing of the margin call stock loan are obtained, and then, the unconditionally stable numerical scheme for the linear complementarity problem is given. And through numerical experiments, we verify the nature of the margin call stock loan, especially when the recourse share $\Delta=0$, and the margin call stock loan is equivalent to the stock loan without recourse. Finally, we give the influence of different parameters on the stock loan with recourse through numerical experiments, especially the influence of different recourse shares $\Delta$, which are unique to the stock loan with recourse, and explain the economic significance behind it.

\section{Data Availability}

The numerical simulation data used to support the findings of this study are included within the article.

\section{Conflicts of Interest}

The authors declare that there are no conflicts of interest regarding the publication of this paper.

\section{Acknowledgments}

The work in this paper was supported by the Ministry of Education of China Project of Humanities and Social Science (No. 19YJA790094), the National Natural Science Foundation of China (No. 11801463), and the Fundamental Research Funds for the Central Universities (JBK2003005).

\section{References}

[1] J. Xia and X. Y. Zhou, "Stock loans," Mathematical Finance, vol. 17, no. 2, pp. 307-317, 2007.

[2] Z. Liang, W. Wu, and S. Jiang, "Stock loan with automatic termination clause, cap, and margin," Computers \& Mathematics with Applications, vol. 60, no. 12, pp. 3160-3176, 2010.

[3] M. R. Grasselli and C. Gómez, "Stock loans in incomplete markets," Applied Mathematical Finance, vol. 20, no. 2, pp. 118-136, 2013.

[4] T. W. Wong and H. Y. Wong, "Valuation of stock loans using exponential phase-type Lévy models," Applied Mathematics and Computation, vol. 222, pp. 275-289, 2013.

[5] N. Cai and L. Sun, "Valuation of stock loans with jump risk," Journal of Economic Dynamics and Control, vol. 40, pp. 213-241, 2014.

[6] T. W. Wong and H. Y. Wong, "Stochastic volatility asymptotics of stock loans: valuation and optimal stopping," Journal of Mathematical Analysis and Applications, vol. 394, no. 1, pp. 337-346, 2012.

[7] P. Carr and L. Wu, "The finite moment log stable process and option pricing," The Journal of Finance, vol. 58, no. 2, pp. 753-777, 2003.
[8] W. Yu, P. Guo, Q. Wang et al., "On a periodic capital injection and barrier dividend strategy in the compound Poisson risk model," Mathematics, vol. 8, no. 4, p. 511, 2020.

[9] W. Yu, F. Wang, Y. Huang, and H. Liu, "Social optimal mean field control problem for the population growth model," Asian Journal of Control, pp. 1-8, 2019.

[10] Z. Zhang, Y. Yong, and W. Yu, "Valuing equity-linked death benefits in general exponential Lévy models," Journal of Computational and Applied Mathematics, vol. 365, Article ID 112377, 2020.

[11] W. Yu, Y. Yong, G. Guan, Y. Huang, W. Su, and C. Cui, "Valuing guaranteed minimum death benefits by cosine series expansion," Mathematics, vol. 7, no. 9, p. 835, 2019.

[12] X. Peng, W. Su, W. Su, and Z. Zhang, "On a perturbed compound Poisson risk model under a periodic thresholdtype dividend strategy," Journal of Industrial \& Management Optimization, vol. 16, no. 4, pp. 1967-1986, 2020.

[13] Á. Cartea, "Dynamic hedging of financial instruments when the underlying follows a non-Gaussian process," SSRN Electronic Journal, 2005.

[14] W. Chen, X. Xu, and S.-P. Zhu, "Analytically pricing European-style options under the modified black-scholes equation with a spatial-fractional derivative," Quarterly of Applied Mathematics, vol. 72, no. 3, pp. 597-611, 2014.

[15] W. Chen, X. Xu, and S.-P. Zhu, "A predictor-corrector approach for pricing American options under the finite moment log-stable model," Applied Numerical Mathematics, vol. 97, pp. 15-29, 2015.

[16] H. Zhang, F. Liu, I. Turner, and S. Chen, "The numerical simulation of the tempered fractional Black-Scholes equation for European double barrier option," Applied Mathematical Modelling, vol. 40, no. 11-12, pp. 5819-5834, 2016.

[17] W. Chen, Numerical methods for fractional Black-Scholes equations and variational inequalities governing option pricing, Ph.D. thesis, The University of Western, London, Canada, 2014.

[18] X. Lu and E. R. M. Putri, "Finite maturity margin call stock loans," Operations Research Letters, vol. 44, no. 1, pp. 12-18, 2016.

[19] B. L. Guo, X. K. Pu, F. H. Huang, Fractional Partial Differential Equations and Their Numerical Solutions, 2015.

[20] Y. L. Zhu, Derivative Securities and Difference Methods, Springer, Berlin, Germany, 2004. 